\title{
Weitere Stabilisierung von Übergewicht und Adipositas
}

Kaspar Staub, Frank Rühli

Danksagung

Die Autoren danken der

Logistikbasis der Schweizer

Armee - Sanität (Oberfeldarzt

Dr. med. A. Stettbacher, Dr. med.

F. Frey) für die Bereitstellung der

Daten, sowie Radoslav Panczak,

Marcel Zwahlen, Ulrich Woitek,

Joel Floris, Christian Pfister und Tobias Schoch für die bisherige Zusammenarbeit. Unsere Studien werden/wurden unterstützt durch die Mäxi Stiftung, die Winkelried Stiftung, das Bundesamt für Gesundheit, den Schweizerischen Nationalfonds und die Stiftung zur Ernährungsforschung in der Schweiz.
Korrespondenz:

Dr. Kaspar Staub

Institut für Evolutionäre

Medizin (IEM)

Universität Zürich

Winterthurerstrasse 190

CH-8057 Zürich

kaspar.staub[at]iem.uzh.ch
Erhöhtes Übergewicht und besonders Adipositas sind mit einem erhöhten Krankheits- und Sterberisiko verbunden und stellen heute ein bedeutendes Gesundheitsproblem dar. Gerade Adipositas wird mit chronischen Stoffwechsel- und Herzkreislauferkrankungen wie Diabetes Typ 2, Bluthochdruck und anderen kardiovaskulären Krankheiten auch schon in jüngeren Altersjahren in Verbindung gebracht. Auch in der Schweiz haben Übergewicht und Adipositas seit Beginn der 1990er-Jahre stark zugenommen. Kürzlich wurde gezeigt, dass in der Schweiz insgesamt 27000 Fälle von Diabetes Typ 2, 63000 Fälle von Bluthochdruck und 37000 Fälle von Dyslipidämie hätten vermieden werden können, wenn das Niveau von Übergewicht und Adipositas auf dem Level von 1992 verblieben wäre. Generell gibt es in der Schweiz allerdings nur wenige landesweite, gemessene und somit objektive, präzise und repräsentative Informationen zur Übergewichtsprävalenz. Ein Monitoring der Übergewichtsprävalenz gerade bei jungen Männern ist dabei gesundheitspolitisch durchaus relevant, da übergewichtige junge Erwachsene das Übergewicht ins Erwachsenenalter weiter mittragen und besonders Männer später einem erhöhten Morbiditäts- und Mortalitätsrisiko ausgesetzt sind.

\section{«Die BMI-Werte scheinen seit drei bis vier Jahren auf konstant hohem Niveau zu verharren.»}

Die gesamtschweizerischen Körpermessdaten der Stellungspflichtigen anlässlich der medizinischen Untersuchung bei der Rekrutierung sind repräsentativ für mindestens $90 \%$ eines Geburtsjahrgangs. Bezogen auf das Individuum kann der Body Mass Index (BMI) grundsätzlich nicht unterscheiden, ob ein Mehr an BMI durch ein Mehr an Muskeloder Fettmasse bestimmt ist. Für Populationsstudien ist der BMI aber durchaus geeignet: Er ist (trotz individueller Ausnahmen, besonders bei Kraftsportlern) auf der Ebene von Gesellschaften stark korreliert mit dem Körperfettanteil und ist das einzige Mass, das bei Datensätzen der vorliegenden Grössenordnung einfach und verlässlich zu messen und zu berechnen

\section{L'IMC des conscrits suisses en}

\section{3: poursuite de la stabilisation du surpoids et de l'obésité}

En Suisse aussi, le surpoids et l'obésité ont gagné du terrain. Sachant que le surpoids persiste à l'âge adulte et que les jeunes hommes en surpoids présentent souvent un risque accru de morbidité et de mortalité une fois adultes, nous avons souhaité étudier la prévalence du surpoids chez ces derniers dans le cadre d'un monitorage représentatif. L'objectif de la présente étude descriptive a consisté à analyser l'indice de masse corporel (IMC) sur la base des données de 2013 relatives aux conscrits suisses au plan national (ce qui représente plus de $\mathbf{9 0} \%$ des jeunes hommes nés au cours de l'année concernée par le recrutement). Les données portent sur 35697 conscrits, dont une majorité âgée de 19 ans, qui mesure en moyenne $178,2 \mathrm{~cm}$ pour $74,3 \mathrm{~kg}$, soit un IMC moyen de $23,4 \mathrm{~kg} / \mathrm{m}^{2}$. La prévalence du surpoids (IMC $25,0-29,9 \mathrm{~kg} / \mathrm{m}^{2}$ ) est de $18,8 \%$ chez les jeunes hommes de 19 ans, et celle de l'obésité (IMC $\geq 30,0 \mathrm{~kg} / \mathrm{m}^{2}$ ) de $5,8 \%$. Compte tenu de ces chiffres, il semblerait que la stabilisation de l'épidémie d'obésité se poursuive dans tous les groupes d'âges étudiés des conscrits de 2013, puisque les valeurs n'ont plus augmenté depuis 3 ou 4 ans. Cependant, ni les causes de cette évolution ni sa progression ultérieure ne sont connues. II est tout à fait possible que les campagnes de prévention intensives menées ces dernières années dans le domaine de la santé publique commencent à déployer leurs effets.

ist. Die Betrachtung von längerfristigen Trends von Körpermassen auf Basis einer gleichbleibenden Datenquelle kann wertvolle Informationen liefern zur Veränderung des Ernährungsstatus und der gesundheitlichen Situation über die Zeit. 2013 wurden die Body-Mass-Index-Daten der Rekrutierung durch das Zentrum für Evolutionäre Medizin (heute Institut für Evolutionäre Medizin) der Universität Zürich 
und das Bundesamt für Gesundheit (BAG) in das Monitoring System für Ernährung und Bewegung (MOSEB) integriert. Im Zuge dieser Forschungen konnten neben regionalen und sozioökonomischen Unterschieden für die Daten von 2004-2012 erstmals gezeigt werden, dass 2010-2012 die BMI-Werte der Stellungspflichtigen nicht mehr zunahmen, also eine Stabilisierung der Übergewichtsepidemie einzusetzen scheint.

Im Zuge der allgemeinen Wehrpflicht werden alle Schweizer Männer, die in einem entsprechenden Jahr ihr 19. Lebensjahr vollenden, zur Rekrutierung aufgeboten. Ebenfalls stellen sich ältere Stellungspflichtige bis zur Vollendung des 25. Altersjahres, welche die Rekrutierung verlegt haben, sowie 18-Jährige, welche die Rekrutierung vorverlegen möchten. Untersuchungen haben gezeigt, dass mindestens $90 \%$ eines männlichen Geburtsjahrgangs im Alter zwischen 18 und 22 Jahren zur Rekrutierung erscheinen. Im Jahre 2004 wurde die Rekrutierung erweitert, sie dauert nun drei Tage und findet nach einheitlichen Standards ganzjährlich in sechs regionalen Rekrutierungszentren (Lausanne VD, Sumiswald BE, Mt. Ceneri TI, Windisch AG, Rüti ZH, Mels SG) statt. Die Ermittlung des Leistungsprofils der Stellungspflichtigen umfasst auch die medizinische Untersuchung des Gesundheitszustandes der jungen Männer, ungeachtet dessen, ob sie später für militärdiensttauglich oder nicht befunden werden. Dabei wird unter anderem auch der BMI berechnet, dafür werden die Stellungspflichtigen ohne Schuhe und in Unterwäsche durch medizinisch geschultes Personal mittels eines Anthropometers in der Körpergrösse vermessen und mittels einer in allen Rekrutierungszentren identischen und von der Armeeapotheke gelieferten und regelmässig geeichten Körperwaage gewogen. Die
BMI-Daten der Stellungspflichtigen stellen aufgrund ihrer Repräsentativität und der gleichbleibenden Erhebungsstandards eine sehr gute Basis für ein kontinuierliches Übergewicht-Monitoring dar.

Der in Tabelle 1 gezeigte Datensatz des Rekrutierungsjahres 2013 umfasst insgesamt N = 35697 Stellungspflichtige. Im Jahre 2013 war die zahlenmässig grösste Altersgruppe der 19-jährigen Stellungspflichtigen im Durchschnitt $178,2 \mathrm{~cm}$ gross (2012: $178,3 \mathrm{~cm}$ ) und 74,3 kg schwer (2012: 74,6 kg). Der mittlere BMI betrug 23,4 kg/m² (2012: 23,5 kg/m²).

\section{«Die Prävalenz von Übergewicht belief sich 2013 bei den 19-Jähri- gen auf 18,8 Prozent.»}

Auch 2013 waren die Verteilungen des Körpergewichts und des BMI im Gegensatz zur Körpergrösse deutlich rechtsschief, höhere Werte waren somit übervertreten. Die Prävalenz von Übergewicht (BMI 25,0$29,9 \mathrm{~kg} / \mathrm{m}^{2}$ ) belief sich 2013 bei den 19-Jährigen auf 18,8 Prozent (2012: 19,6 Prozent), die Prävalenz von Adipositas (BMI $\geq 30,0 \mathrm{~kg} / \mathrm{m}^{2}$ ) betrug 5,8 Prozent (2012: 5,9 Prozent). Insgesamt hatten somit auch 2013 24,7 Prozent (2012: 25,5 Prozent) einen BMI von mindestens $25,0 \mathrm{~kg} / \mathrm{m}^{2}$ und waren damit übergewichtig. Auch in der jüngeren und älteren Alterskategorie unterscheiden sich die Mittelwerte und Prävalenzen für das Jahr 2013 kaum von den Werten für die Jahre 2010-2012. Das Rekrutierungsjahr 2013 stellt somit sowohl beim mittleren BMI der 19-jährigen Stellungspflichtigen seit 1952 als auch bei den

Tabelle 1

Körpergrösse, Gewicht, Body Mass Index (BMI) sowie die Prävalenzen von Übergewicht und Adipositas bei 18-jährigen, 19-jährigen und 20-jährigen Schweizer Stellungspflichtigen im Rekrutierungsjahr 2013.

\begin{tabular}{|c|c|c|c|c|c|c|c|c|c|}
\hline & \multicolumn{3}{|c|}{$[18-19)$} & \multicolumn{3}{|c|}{$[19-20)$} & \multicolumn{3}{|c|}{$[20-21)$} \\
\hline & $\begin{array}{l}\text { Länge } \\
(\mathrm{cm})\end{array}$ & $\begin{array}{l}\text { Gewicht } \\
\text { (kg) }\end{array}$ & $\begin{array}{c}\text { BMI } \\
\left(\mathrm{kg} / \mathrm{m}^{2}\right)\end{array}$ & $\begin{array}{l}\text { Länge } \\
(\mathrm{cm})\end{array}$ & $\begin{array}{l}\text { Gewicht } \\
\text { (kg) }\end{array}$ & $\begin{array}{c}\text { BMI } \\
\left(\mathrm{kg} / \mathrm{m}^{2}\right)\end{array}$ & $\begin{array}{l}\text { Länge } \\
(\mathrm{cm})\end{array}$ & $\begin{array}{l}\text { Gewicht } \\
\text { (kg) }\end{array}$ & $\begin{array}{c}\text { BMI } \\
\left(\mathrm{kg} / \mathrm{m}^{2}\right)\end{array}$ \\
\hline$N$ & 8624 & 8624 & 8624 & 15452 & 15452 & 15452 & 7171 & 7171 & 7171 \\
\hline Mittelwert & 178,43 & 73,39 & 23,03 & 178,21 & 74,28 & 23,36 & 177,99 & 75,14 & 23,69 \\
\hline Standardabweichung & 6,58 & 12,69 & 3,62 & 6,53 & 13,03 & 3,74 & 6,55 & 13,74 & 3,99 \\
\hline Standardfehler Mittelwert & 0,071 & 0,137 & 0,039 & 0,053 & 0,105 & 0,030 & 0,077 & 0,162 & 0,047 \\
\hline Median & 178,0 & 71,0 & 22,3 & 178,0 & 72,0 & 22,7 & 178,0 & 73,0 & 23,0 \\
\hline Minimum & 153,0 & 41,0 & 14,8 & 150,0 & 41,0 & 14,5 & 150,0 & 41,0 & 14,7 \\
\hline Maximum & 207,0 & 176,0 & 48,2 & 208,0 & 178,0 & 57,5 & 203,0 & 174,0 & 52,2 \\
\hline \multirow[t]{2}{*}{ Schiefe } & 0,10 & 1,40 & 1,56 & 0,09 & 1,30 & 1,50 & 0,11 & 1,40 & 1,57 \\
\hline & $\mathrm{N}$ & $\%$ & & $\mathrm{~N}$ & $\%$ & & $N$ & $\%$ & \\
\hline $\mathrm{BMI}<18,5$ & 380 & $4,4 \%$ & & 622 & $4,0 \%$ & & 246 & $3,4 \%$ & \\
\hline BMI 18,5-24,9 & 6387 & $74,1 \%$ & & 11034 & $71,4 \%$ & & 4914 & $68,5 \%$ & \\
\hline BMI 25,0-29,9 & 1428 & $16,6 \%$ & & 2899 & $18,8 \%$ & & 1527 & $21,3 \%$ & \\
\hline $\mathrm{BMI} \geq 30,0$ & 429 & $5,0 \%$ & & 897 & $5,8 \%$ & & 484 & $6,7 \%$ & \\
\hline
\end{tabular}




\section{Abbildung 1}

Der mittlere BMI der 19-jährigen Stellungspflichtigen 1952-2013 sowie der 18- und 20-jährigen jungen Männer 2004-2013 (rechts = neueste Daten des Jahres 2013).

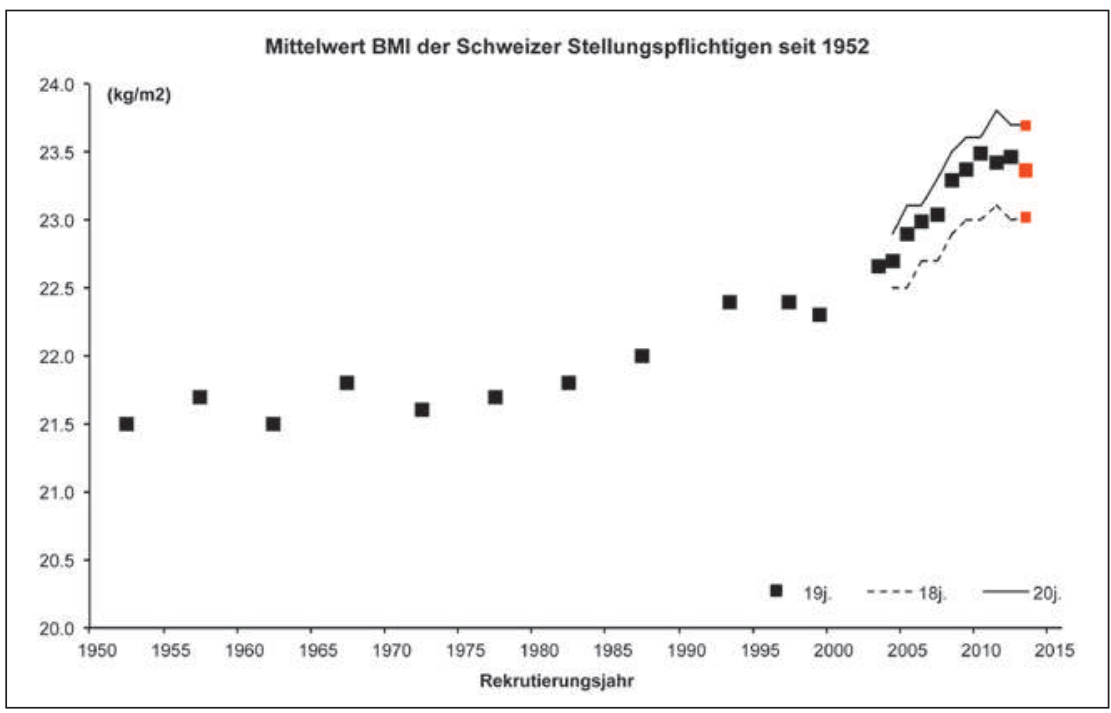

Abbildung 2

Die Häufigkeiten (Prävalenz) von Übergewicht (BMI 25,0-29,9 kg/m²) und Adipositas (BMI $\geq 30,0 \mathrm{~kg} / \mathrm{m}^{2}$ ) bei der zahlenmässig grössten Altersgruppe der 19-jährigen Stellungspflichtigen 2004-2013. Rechts $=$ Neueste Daten des Rekrutierungsjahres 2013, Fehlerbalken = 95\%-Konfidenzintervall.

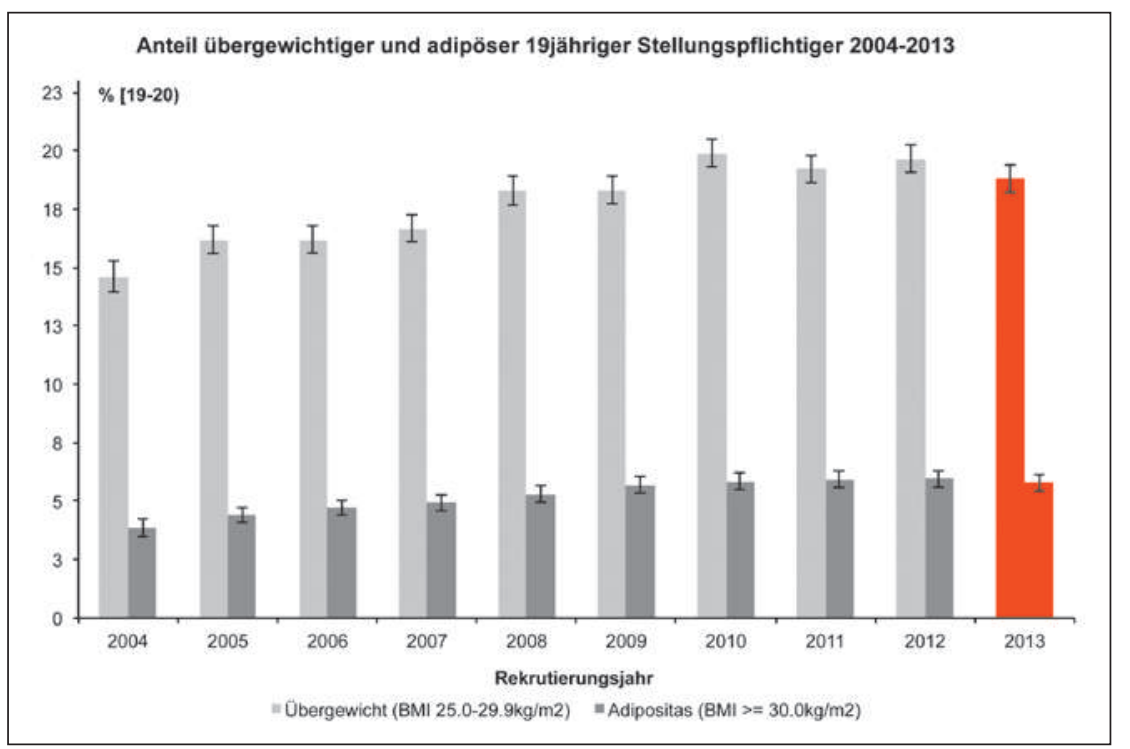

anderen Altersgruppen seit 2004 nach 2011 und 2012 ein weiteres Jahr der Stabilisierung dar (Abb. 1).

Auch die Prävalenzen für Übergewicht (BMI $25,0-29,9 \mathrm{~kg} / \mathrm{m}^{2}$ ) und Adipositas (BMI $\left.\geq 30,0 \mathrm{~kg} / \mathrm{m}^{2}\right)$ sind 2013 nicht weiter angestiegen, wie am Beispiel der 19-jährigen Männer in Abbildung 2 zu sehen ist. Die BMI-Werte, die seit Ende der 1980er Jahre besonders stark zugenommen hatten, scheinen nunmehr seit drei bis vier Jahren auf konstant hohem Niveau zu verharren. Diese Beobachtung deckt sich gut mit Daten von Schulkinderuntersuchungen, wo ebenfalls seit einigen Jahren eine Plafonierung festgestellt wird.
Über die Ursachen dieser Entwicklung und den weiteren Fortgang herrscht nach wie vor Unklarheit. Es ist möglich, dass die in den letzten Jahren intensivierten Präventionskampagnen im Public HealthBereich jetzt Wirkung zeigen. Zum Beispiel zeigen die Auswertungen der Leistungen beim Test Fitness Rekrutierung (TFR) durch das Bundesamt für Sport seit 2006, dass sich die Ausdauerleistung der Stellungspflichtigen (nach vorheriger stetiger Abnahme) seit 2006 konstant verhält. Damit hat sich offenbar vor der Stabilisation des BMI bereits das Bewegungsverhalten der jungen Männer verändert. Ob sich das Ernährungsverhalten der jungen Männer ebenfalls entspre-

\section{Jeder vierte Stellungspflichtige war übergewichtig oder adipös.}

chend verändert hat, müsste eingehender untersucht werden. Als Schwäche der Stellungspflichtigen-Daten muss festgehalten werden, dass bei BMI-basierten Studien zu den Stellungspflichtigen gerade im leicht übergewichtigen Bereich (BMI bis ca. $27-28 \mathrm{~kg} / \mathrm{m}^{2}$ ) bei intensiv Kraftsportarten ausübenden jungen Männern durchaus Fehleinschätzungen betreffend Fett- oder Muskelmasse möglich sind. Im stark übergewichtigen oder gar adipösen Bereich werden sich diese Fehleinschätzungen aber auf ein Minimum beschränken. Weitere Untersuchungen müssten daher den Gesamtkörperfettanteil der jungen Männer nicht nur anhand des BMI schätzen, sondern ebenfalls bspw. mittels Waist Circumference die für das Krankheits- und Sterberisiko noch wichtigere Körperfettverteilung miteinbeziehen. Auf jeden Fall muss die zukünftige Entwicklung im Auge behalten werden, da auch 2013 nach wie vor rund 25 Prozent und damit jeder vierte Stellungspflichtige übergewichtig oder adipös waren. Ob zukünftig im Zuge der intensivierten Public HealthMassnahmen sogar eine Trendwende einsetzt und die Werte zu sinken beginnen, wird erst die Untersuchung der kommenden Jahre zeigen.

\section{Referenzen}

- Staub K, Rühli F. Der Body Mass Index der Schweizer Stellungspflichtigen 2013 im Lichte der historischen Entwicklung. Swiss Review of Military and Disaster Medicine. 2014(2):39-44

- Panczak R, Woitek U, Rühli F, Staub K. Regionale und sozio-ökonomische Unterschiede im Body Mass Index (BMI) von Schweizer Stellungspflichtigen 2004-2012. Projektschlussbericht zuhanden des Bundesamtes für Gesundheit (BAG). Zürich; 2013

- Panczak R, Zwahlen M, Woitek U, Rühli FJ, Staub K. Socioeconomic, temporal and regional variation in Body Mass Index among 188,537 Swiss conscripts born between 1986 and 1992. PLoS One 9: e96721; 2014.

- Weitere Referenzen bei den Autoren. 